Meta

Journal des traducteurs

Translators' Journal

\title{
Les éponymes médicaux : essai de classification
}

\section{Henri Van Hoof}

Volume 31, numéro 1, mars 1986

Traduction et terminologie médicale

Medical Translation and Terminology

URI : https://id.erudit.org/iderudit/004543ar

DOI : https://doi.org/10.7202/004543ar

Aller au sommaire du numéro

Éditeur(s)

Les Presses de l'Université de Montréal

ISSN

0026-0452 (imprimé)

1492-1421 (numérique)

Découvrir la revue

Citer ce document

Van Hoof, H. (1986). Les éponymes médicaux : essai de classification. Meta,

31(1), 59-84. https://doi.org/10.7202/004543ar d'utilisation que vous pouvez consulter en ligne.

https://apropos.erudit.org/fr/usagers/politique-dutilisation/ 


\title{
LES ÉPONYMES MÉDICAUX : ESSAI DE CLASSIFICATION
}

\author{
HENRI VAN HOOF
}

\begin{abstract}
L'habitude d'accoler un éponyme à une loi, à une maladie, à un symptôme ou à une unité est fort ancienne ; on a ainsi perpétué la mémoire de ceux qui ont contribué à la science ou à l'art de guérir, et c'est justice.
\end{abstract}

Ainsi s'exprimait le Dr A. Sliosberg dans un article sur la traduction médicale et pharmaceutique ${ }^{1}$. Il ajoutait toutefois, un peu plus loin, que " le nationalisme aidant, de telles désignations perdent de leur universalité et peuvent embarrasser le lecteur ». De fait, dans plusieurs de nos publications consacrées aux problèmes de la traduction médico-pharmaceutique ${ }^{2,3,4}$, nous avons eu l'occasion d'étudier quelques-unes des difficultés soulevées par la traduction des éponymes.

Les éponymes médicaux sont de deux types selon que le nom propre a été banalisé (parkinsonism/parkinsonisme) ou est demeuré nom propre (Broca's amnesia/amnésie de Broca). Déjà les noms communs réservent quelques surprises, car la forme qu'ils revêtent dans une langue n'existe pas toujours ou n'est pas nécessairement identique dans l'autre. L'emploi des noms propres tels quels ne simplifie pas le problème pour autant. Tout d'abord, parce qu'ils sont beaucoup plus nombreux, ensuite, parce que leur traduction ne semble répondre à aucune règle. Un terme anglais construit avec un épon.yme peut très bien avoir un équivalent français sans éponyme et vice versa, ou à un éponyme anglais peut très bien correspondre un autre éponyme français, ou l'éponyme peut être identique dans les deux langues mais accompagner un déterminé différent, etc. Enfin, le casse-tête s'aggrave encore lorsque plusieurs éponymes - et pas toujours les mêmes dans les deux langues - servent à désigner un même concept.

Pour essayer d'y voir un peu plus clair, nous avons eu la curiosité d'examiner si les éponymes médicaux se prêtaient à un essai de classification pour la combinaison anglais-français.

\section{1. ÉPONYMES IDENTIQUES DANS LES DEUX LANGUES}

\subsection{EPONYMES ANGLAIS CONSTRUITS AVEC LE GÉNITIF SAXON}

\subsubsection{Formes simples}

La grande majorité des éponymes médicaux anglais sont du type éponyme simple + génitif saxon : Corti's organ, Meckel's diverticulum, etc. Il arrive cependant que la marque du génitif disparaisse : Coolidge tube, Edward syndrome, Ehrlich granules, Foley catheter, Grice procedure, Haverhill fever, Michel clip, Pick cell, Sandstroem glands, Tyndall phenomenon, Urisberg cartilage, etc. Lorsque l'éponyme se termine par la lettre $s, s c h, x$ ou $z$, la règle voudrait qu'il ne prenne que l'apostrope du génitif : Colles' ligament, Deiters' process, etc. Si cette règle se vérifie généralement pour la lettre $s$ (bien qu'elle aussi connaisse des exceptions : Bence Jones protein, Bywaters syndrome, Denonvilliers fascia, Jacobäus operation, Verres needle, etc. sans apostrophe ; Bross's sequential 
scheme, Capgras's syndrome, Miles's disease, etc. avec $s$ ajouté), il y est dérogé avec constance pour sch, $x, z$ : Andersch's ganglion, Darkschewitsch's nucleus, Spix's spine, Rieux's syndrome, Valleix's spots, Bogomoletz's serum, Foltz's valve, Grawitz's granule, Mickulicz's cells, Opitz's syndrome, Treitz's hernia, Vaguez's disease, etc.

\begin{tabular}{|c|c|c|}
\hline Albert's operation & Opération d'Albert & Gaucher's cells \\
\hline Andersch's ganglion & Ganglion d'Andersch & Gennari's line \\
\hline Aschoff's nodules & Nodes d'Aschoff & Gerlach's network \\
\hline Auerbach's plexus & Plexus d'Auerbach & Giacomini's band \\
\hline Babinski's sign & Signe de Babinski & Gilchrist's disease \\
\hline Baillerger's strips & Stries de Baillarger & Glisson's capsule \\
\hline Bechterew's nucleus & Noyau de Bechterew & Golgi's apparatus \\
\hline Bochdalek's duct & Canal de Bochdalek & Grawitz's granules \\
\hline Bogomoletz's serum & Sérum de Bogomoletz & Günther's disease \\
\hline Bouchard's nodes & Nodosités de Bouchard & Haller's aberrant duct \\
\hline Brill's disease & Maladie de Brill & Harley's disease \\
\hline Brissaud's infantilism & Infantilisme de Brissaud & Haverhill fever \\
\hline Broca's amnesia & Amnésie de Broca & Hebra's prurigo \\
\hline Burdach's tract & Faisceau de Burdach & Heidenhain's cells \\
\hline Bywaters syndrome & Syndrome de Bywaters & Henle's loop \\
\hline Capgras's syndrome & Syndrome de Capgras & Heschl's gyrus \\
\hline Castle's factor & Facteur de Castle & Highmore's body \\
\hline Charcot's signe & Signe de Charcot & Hirschsprung's disease \\
\hline Civinni's process & Epine de Civinni & Hortega cells \\
\hline Claudius' fossa & Fossette de Claudius & Houston's valves \\
\hline Cloquet's hernia & Hernie de Cloquet & Hunter's canal \\
\hline Colles' ligament & Ligament de Colles & Huntington's chorea \\
\hline Coolidge tube & Tube de Coolidge & Hutchinson's teeth \\
\hline Corrigan's pulse & Pouls de Corrigan & Huxley's layer \\
\hline Corti's membrane & Membrane de Corti & Jackson's membrane \\
\hline Corvisart's facies & Faciès de Corvisart & Jacobäus operation \\
\hline Cowper's gland & Glande de Cowper & Jacobson's organ \\
\hline Crohn's disease & Maladie de Crohn & Kennedy's syndrome \\
\hline Cushing's syndrome & Syndrome de Cushing & Kienböck's phenomenon \\
\hline Danlos' syndrome & Syndrome de Danlos & Kiernan's space \\
\hline Darkschewitsch's nucleus & Noyau de Darkschewitsch & Korsakoff's psychosis \\
\hline Deiters' process & Prolongement de Deiters & Krause's line \\
\hline Döderlein's bacillus & Bacille de Döderlein & Krönlein's hernia \\
\hline Donovan's bodies & Corps de Donovan & Krukenberg's tumor \\
\hline Ebner's glands & Glandes d'Ebner & Kühne's terminal plate \\
\hline Emmet's operation & Opération d'Emmet & Kupffer's cells \\
\hline Ewing's sarcoma & Sarcome d'Ewing & Kussmaul's disease \\
\hline Felty's syndrome & Syndrome de Felty & Laennec's cirrhosis \\
\hline Flechsig's tract & Faisceau de Flechsig & Langer's axillary arch \\
\hline Flexner's bacillus & Bacille de Flexner & Langhans' layer \\
\hline Foltz's valve & Valvule de Foltz & Larrey's cleft \\
\hline Fontana's spaces & Espaces de Fontana & Lee's ganglion \\
\hline Froment's paper sign & $\begin{array}{l}\text { Signe du journal de Fro- } \\
\text { ment }\end{array}$ & $\begin{array}{l}\text { Le Fort's fracture } \\
\text { Leishman's nodules }\end{array}$ \\
\hline Gartner's & Canal de Gartner & Lieberkühn's glands \\
\hline
\end{tabular}

\author{
Cellules de Gaucher \\ Raie de Gennari \\ Réseau de Gerlach \\ Bandelette de Giacomini \\ Maladie de Gilchrist \\ Capsule de Glisson \\ Appareil de Golgi \\ Granulations de Grawitz \\ Maladie de Günther \\ Vas aberrans de Haller \\ Maladie de Harley \\ Fièvre de Haverhill \\ Prurigo de Hebra \\ Cellules de Heidenhain \\ Anse de Henle \\ Circonvolution de Heschl \\ Corps de Highmore \\ Maladie de Hirschsprung \\ Cellules d'Hortega \\ Valvules de Houston \\ Canal de Hunter \\ Chorée de Huntington \\ Dents de Hutchinson \\ Couche de Huxley \\ Membrane de Jackson \\ Općration de Jacobäus \\ Organe de Jacobson \\ Syndrome de Kennedy \\ Phénomène de Kienböck \\ Espace de Kiernan \\ Psychose de Korsakoff \\ Strie de Krause \\ Hernie de Krönlein \\ Tumeur de Krukenberg \\ Plaque terminale de Kühne \\ Cellules de Kupffer \\ Maladie de Kussmaul \\ Cirrhose de Laennec \\ Arc axillaire de Langer \\ Couche de Langhans \\ Fente de Larrey \\ Ganglion de Lee \\ Fracture de Le Fort \\ Nodules de Leishman \\ Glandes de Lieberkühn
}




\begin{tabular}{l} 
Lisfranc's joint \\
Lissauer's marginal zone \\
Lobstein's cancer \\
Louis' angle \\
Ludwig's angina \\
Luschka's tonsil \\
Malacarne's pyramid \\
Mandelbaum's reaction \\
Marfan's disease \\
McBurney's point \\
Meckel's diverticulum \\
Meissner's corpuscles \\
Meynert's bundle \\
Mickulicz's cells \\
Moebius' disease \\
Moeller's glossitis \\
Montgomery's tubercles \\
Morgagni's lacunae \\
Moro's reflex \\
Morvan's chorea \\
Mïller's fibres \\
Murphy's method \\
Nägele's pelvis \\
Negri's bodies \\
Nelaton's catheter \\
Nettleship's disease \\
Neumann's sheath \\
Nissl's degeneration \\
Obermeier's spirillum \\
Opitz's syndrome \\
Osler's nodule \\
Paget's disease \\
Parrot's pseudoparalysis \\
Parinaud's conjunctivitis \\
Peyer's patches \\
Pfeiffer's bacillus \\
Pick's syndrome \\
Purkinje's cells \\
Queckenstedt's signe \\
Ranvier's constriction \\
Reissner's membrane \\
Remak's ganglion \\
Reverdin's graft \\
Ridley's sinus \\
Riedel's lobe \\
Rieux's hernia \\
Robert's pelvis \\
Romberg's sign \\
Rosenmüller's fossa \\
Rous' sarcoma \\
\\
\hline
\end{tabular}

Articulation de Lisfranc Zone marginale de Lissauer Cancer de Lobstein Angle de Louis Angine de Ludwig Amygdale de Luschka Pyramide de Malacarne Réaction de Mandelbaum Maladie de Marfan Point de McBurney Diverticule de Meckel Corpuscules de Meissner Faisceau de Meynert Cellules de Mickulicz Maladie de Moebius Glossite de Moelier Tubercules de Montgomery Lacunes de Morgagni Réflexe de Moro Chorée de Morvan Fibres de Müller Méthode de Murphy Bassin de Nägele Corps de Negri Cathéter de Nélaton Maladie de Nettleship Gaine de Neumann Dégénérescence de Nissl Spirochète d'Obermeier Syndrome d'Opitz Nodule d'Osler Maladie de Paget Pseudoparalysie de Parrot Conjonctivite de Parinaud Plaques de Peyer Bacille de Pfeiffer Syndrome de Pick Cellules de Purkinje Signe de Queckenstedt Étranglement de Ranvier Membrane de Reissner Ganglion de Remak Greffe de Reverdin Sinus de Ridley Lobe de Riedel Hernie de Rieux Bassin de Robert Signe de Romberg Fossette de Rosenmiller Sarcome de Rous
Ruffini's corpuscles Russell's bodies Santorini's cartilage Scarpa's triangle Schlemm's canal Schridde's granules Shenton's line Shrapnell's membrane Simmonds' disease Spix's spine Sprengel's deformity Stiller's sign Stilling's sacral nucleus Sudeck's anastomosis Sutton's disease Talma's operation Tardieu's spots

Tenon's capsule

Thomsen's disease

Tinel's sign

Todd's paralysis

Tomes' granular layer

Trantas' dots

Traube's semilunar space

Treitz's hernia

Tuffier's operation

Tyndall phenomenon

Uskow's pillars

Valleix's points

Vaquez's disease

Verga's ventricle

Vicq d'Azyr's band

Vieussens' annulus

Virchow's cells

Volkmann's deformity

Walther's ducts

Wegener's granulomatosis

Werlhof's disease

Wernicke's aphasia

Wharton's duct

Whipple's disease

Willard's lupus

Willis' cords

Woillez's disease

Wrisberg's ganglion

Young's syndrome

Zaglas' ligament

Zinn's circle

Zuckerkandl's gland
Corpuscules de Ruffini Corps de Russell Cartilage de Santorini Triangle de Scarpa Canal de Schlemm Granules de Schridde Ligne de Shenton Membrane de Shrapnell Maladie de Simmonds Epine de Spix Déformation de Sprengel Signe de Stiller Noyau sacré de Stilling Anastomose de Sudeck Maladie de Sutton Opération de Talma Taches de Tardieu Capsule de Tenon Maladie de Thomsen Signe de Tinel Paralysie de Todd Couche granulaire de Tomes

Granules de Trantas

Espace semilunaire de Traube

Hernie de Treitz Opération de Tuffier Phénomène de Tyndall Piliers d'Uskow

Points de Valleix Maladie de Vaquez Ventricule de Verga Ruban de Vicq d'Azyr Anneau de Vieussens Cellules de Virchow Difformité de Volkmann Canaux de Walther Granulomatose de Wegener Maladie de Werlhof Aphasie de Wernicke Canal de Wharton Maladie de Whipple Lupus de Willard Cordes de Willis Maladie de Woillez Ganglion de Wrisberg Syndrome de Young Lgament de Zaglas Cercle de Zinn Glande de Zuckerkandl 


\subsubsection{Formes composées}

Les éponymes composés sont généralement du type éponyme + éponyme sans la marque du génitif.

Albright-McCune-Sternberg syndrome

Aschheim-Zondek test

Brill-Symmers disease

Busse-Buschke disease

Calmette-Guérin bacillus

Charcot-Leyden crystals

Charcot-Weits-Barber syndrome

Cheyne-Stokes respiration

Cruveilhier-Baumgarten cirrhosis

Déjerine-Roussy syndrome

Déjerine-Sottas disease

Déjerine-Thomas syndrome

Ehlers-Danlos disease

Féréol-Graux ocular palsy

Fanconi-Patrassi syndrome

Foix-Alajouanine syndrome

Guillain-Barré syndrome

Heine-Medin disease

Jaffe-Liechtenstein disease

Kashin-Beck disease

Key-Retzius foramen

Koch-Weeks bacillus

Läwen-Roth syndrome

Lutz-Splendon-Almeida disease

MacClure-Aldrich test

Marchiafava-Bignami disease

Merzbacher-Pelizaus disease

Osler-Vaquez disease

Raymond-Cesnan syndrome

Riley-Day syndrome

Riva-Rocci sphygmomanometer

Roussy-Levy disease

Senear-Usher disease

Silvestrini-Corda syndrome

Schultz-Charlton phenomenon

Spiegler-Fendt sarcoid

Sulzberger-Garbe disease

Urbach-Wiethe disease

Virchow-Robin space

Vogt-Koyanagi syndrome

Waterhouse-Friderichsen syndrome

Ziehen-Oppenheim disease
Syndrome d'Albright-McCune-Sternberg

Test d'Aschheim-Zondek

Maladie de Brill-Symmers

Maladie de Busse-Buschke

Bacille de Calmette-Guérin

Cristaux de Charcot-Leyden

Syndrome de Charcot-Weits-Barber

Respiration de Cheyne-Stokes

Cirrhose de Cruveilhier-Baumgarten

Syndrome de Déjerine-Roussy

Maladie de Déjerine-Sottas

Syndrome de Déjerine-Thomas

Maladie d'Ehlers-Danlos

Paralysie oculaire de Féréol-Graux

Syndrome de Fanconi-Patrassi

Syndrome de Foix-Alajouanine

Syndrome de Guillain-Barré

Maladie de Heine-Medin

Maladie de Jaffe-Liechtenstein

Maladie de Kashin-Beck

Trou de Key-Retzius

Bacille de Koch-Weeks

Syndrome de Läwen-Roth

Maladie de Lutz-Splendon-Almeida

Test de MacClure-Aldrich

Maladie de Marchiafava-Bignami

Maladie de Merzbacher-Pelizaeus

Maladie d'Osler-Vaquez

Syndrome de Raymond-Cesnan

Syndrome de Riley-Day

Sphygmomanomètre de Riva-Rocci

Maladie de Roussy-Levy

Maladie de Senear-Usher

Syndrome de Silvestrini-Corda

Phénomène de Schultz-Charlton

Sarcoïde de Spiegler-Fendt

Maladie de Sulzberger-Garbe

Maladie d'Urbach-Wiethe

Espace de Virchow-Robin

Syndrome de Vogt-Koyanagi

Syndrome de Waterhouse-Friderichsen

Maladie de Ziehen-Oppenheim

Il existe cependant des exceptions à ce schéma, où l'association éponymique prend la marque du génitif :

André-Thomas' sign
Brown-Séquard's paralysis
Signe d'André-Thomas

Syndrome de Brown-Séquard 
Gombault-Philippe's triangle

Klippel-Feil's syndrome

Pfaundler-Hurler's disease

Strüimpell-Westphal's syndrome
Faisceau de Gombault et Philippe

syndrome de Klippel-Feil

Syndrome de Pfaundler-Hurler

Syndrome de Westphal-Strümpell

\section{2 ÉPONYMES ANGLAIS CONSTRUITS AVEC LE GÉNITIF FRANÇAIS}

À côté de la construction éponymique classique avec 's, l'anglais présente un certain nombre de termes formés sur le modèle du génitif français au moyen de of. Ceci ne signifie toutefois pas que les éponymes rencontrés dans les exemples de ce type ne puissent en d'autres occasions suivre la construction classique.

Nodules of Arantius

Fatty ball of Bichat

Column of Burdach

Canal of Corti

Ducts of Cuvier

Arcuate line of Douglas

Tetralogy of Fallot

Pyramids of Ferrein

Column of Gowers

Rods of Heidenhain

Sheath of Henle

Antrum of Highmore

Muscle of Horner

Nerve of Jacobson

Node of Keith and Flack

Islands of Langerhans

Body of Luys

Pyramid of Malpighi

Fissure of Monro

Columns of Morgagni

Cistern of Pecquet

Isiand of Reil

Fibres of Remak

Fissure of Rolando

Organs of Ruffini

Uncinate fasciculus of Russell

Tubercle of Santorini

System of Sappey

Sheath of Schwann

Fleace of Stilling

Aqueduct of Sylvius

Muscle of Treitz

Sinus of Valsalva

Ampulla of Vater

Stars of Verheyen

Foramen of Vesalius

Bundle of Vicq d'Azyr

Ansa of Vieussens

Foramen of Winslow

Canal of Wirsung
Nodules d'Arantius

Boule graisseuse de Bichat

Faisceau de Burdach

Tunnel de Corti

Sinus de Cuvier

Arcade de Douglas

Pyramides de Fallot

Pyramides de Ferrein

Faisceau de Gowers

Bâtonnets de Heidenhain

Gaine de Henle

Antre de Highmore

Muscle de Horner

Nerf de Jacobson

Nœud de Keith et Flack

Ilôts de Langerhans

Corps de Luys

Pyramide de Malpighi

Sillon de Monro

Colonnes de Morgagni

Citerne de Pecquet

Insula de Reil

Fibres de Remak

Scissure de Rolando

Organes de Ruffini

Faisceau en crochet de Russell

Tubercule de Santorini

Système de Sappey

Gaine de Schwann

Lacis de Stilling

Aqueduc de Sylvius

Muscle de Treitz

Sinus de Valsalva

Ampoule de Vater

Étoiles de Verheyen

Trou de Vésale

Faisceau de Vicq d'Azyr

Anse de Vieussens

Hiatus de Winslow

Canal de Wirsung 
Glands of Zeis

Glandes de Zeis

Organs of Zuckerkandl

Organes de Zuckerkandl

\section{ÉPONYMES PRÉCÉDÉS D'UN PRÉNOM OU D'INITIALES}

\subsubsection{Dans les deux langues}

C. Vogt's syndrome

S.A.K. Wilson's disease

W.J.E. Wilson's disease

\subsubsection{En anglais seulement}

Ch. Abadie's sign

J. Abadie's sign

S. Fothergill's neuralgia

Austin Flint's murmur

\subsubsection{En français seulement}

Nerve of Bell

Guérin's fracture

Petit's ligament

Duverney's gland

Hunt's neuralgia

Müller's sign
Syndrome de C. et O. Vogt

Maladie de S.A.K. Wilson

Maladie de W.J.E. Wilson

Signe d'Abadie

Signe d'Abadie du tabès

Maladie de Fothergill

Signe de Flint

Nerf de Ch. Bell

Fracture d'A. Guérin

Ligament de J.L. Petit

Glande de Joseph du Verney

Névralgie de Ramsey Hunt

Signe de Friedrich von Müller

\section{4 ÉPONYMES ÉTOFFÉS D'UNE PRÉCISION COMPLÉMENTAIRE}

Dans de très nombreux cas, le déterminé de l'éponyme est étoffé d'une précision anatomique, morphologique ou autre.

\subsubsection{Formes simples}

1.4.1.1 Précision complémentaire en anglais

Barré's pyramidal sign

Cooper's suspensory ligament

Döhle's inclusion bodies

Farre's white line

Löwenthal's marginal bundle

Mangoldt's epithelial grafting

Marshall's oblique vein

Nunn's gorged corpuscles

Sattler's elastic layer

Schmorl's necrosis bacillus

Stahli's pigment line

Sternberg's giant cells

Trousseau's meningitic streak
Signe de Barré

Ligament de Cooper

Inclusions de Döhle

Ligne de Farre

Faisceau de Löwenthal

Greffe de Mangoldt

Veine de Marshall

Corpuscules de Nunn

Couche de Sattler

Bacille de Schmorl

Ligne de Stahli

Cellules de Sternberg

Raie de Trousseau

À l'occasion, le terme éponymique est construit avec le génitif français :

Auditory teeth of Huschke

Comma tract of Schultze
Dents de Huschke

Virgule de Schultze 


\subsubsection{Précision complémentaire en français}

Arnold's nerve
Eéclard's nucleus
Eichat's canal
Eoyer's bursa
Eruch's layer
Caldani's ligament
Castellani's bronchitis
Denucé's ligament
Forel's commissure
Gerdy's fontanel
Gillie's graft
Goll's tract
Haller's plexus
Harris' band
Hebra's eczema
Henle's gland
Herxheimer's fibres
Jacobson's retinitis
Knapp's streaks
Laennec's infarctus
Langerhans' cells
Luschke's ligament
Meynert's cells
Monaldi's drainage
Mondanesi's reflex
Paget's cells
Perlia's nucleus
Pick cell
Riedel's disease
Rose's tetanus
RRosenthal's canal
Sonne's dysentery
Staderini's nucleus
Tenon's space
Treitz's arch
Vieussens' veins
Volkmann's contracture
Weinbrecht's cord
Welander's ulcer
Wrisberg's ansa

Nerf jugulaire d'Arnold

Noyau d'ossification de Béclard

Canal arachnoïdien de Bichat

Bourse séreuse de Boyer

Lame vitrée de Bruch

Ligament bicorne de Caldani

Bronchite sanglante de Castellani

Ligament carré de Denucé

Commissure sous-optique de Forel

Fontanelle sagittale de Gerdy

Lambeau tubulé de Gillie

Faisceau grêle de Goll

Plexus laryngé de Haller

Bride péritonéale de Harris

Eczéma marginé de Hebra

Glande tubuleuse de Henle

Fibres spiralées de Herxheimer

Rétinite syphilitique de Jacobson

Stries angioïdes de Knapp

Infarctus hémoptoïque de Laennec

Cellules épidermiques de Langerhans

Ligament sterno-péricardique de Luschke

Cellules pyramidales de Meynert

Drainage intracavitaire de Monaldi

Réflexe facial de Mondanesi

Cellules dyskératosiques de Paget

Centre oculomoteur de Perlia

Cellule spumeuse de Pick

Maladie ligneuse de Riedel

Tétanos céphalique de Rose

Canal spinal de Rosenthal

Dysenterie bacillaire de Sonne

Noyau intercalaire de Staderini

Espace intervaginal de Tenon

Arc vasculaire de Treitz

Veines innominées de Vieussens

Rétraction ischémique de Volkmann

Corde ligamenteuse de Weinbrecht

Ulcère vénéroïde de Welander

Anse mémorable de Wrisberg

De telles constructions se rencontrent aussi, quoique beaucoup plus rarement, à partir d'éponymes adjectivés ou construits avec un génitif français :

Pacchionian foramen

Canal of Arantius

Radiation of Gratiolet
Foramen ovale de Pacchioni

Canal veineux d'Arantius

Radiation optique de Gratiolet 


\subsubsection{Formes composées}

1.4.2.1 Précision complémentaire en anglais Argyll-Robinson pupil sign

Signe d'Argyll-Robinson

1.4.2.2 Précision complémentaire en français

Darier-Roussy sarcoid

Klebs-Löffler bacillus

Fiessinger-Leroy syndrome
Sarcoïde hypodermique de Darier-Roussy

Bacille diphtérique de Klebs-Löffler

Syndrome conjonctivo-urétro-synovial de Fiessinger-Leroy

\subsection{DÉTERMINÉ DIFFÉRENT DANS LES DEUX LANGUES}

Dans de nombreux termes, le même éponyme accompagne un déterminé différent.

\subsubsection{Formes simples}

Parfois, la différence est minime :

Arnaud's syndrome

Behr's disease

Haglund's disease

Heerfordt's disease

Kocher's method

Marfan's method

Gordon's reflex

Schaeffer's reflex

Pietrowski's sign

Prevost's sign

Strümpell's sign

Tournay's sign

\author{
Maladie d'Arnaud \\ Syndrome de Behr \\ Syndrome de Haglund \\ Syndrome de Heerfordt \\ Procédé de Kocher \\ Procédé de Marfan \\ Signe de Gordon \\ Signe de Schaeffer \\ Phénomène de Pietrowski \\ Phénomène de Prévost \\ Phénomène de Strümpell \\ Phénomène de Tournay
}

Dans la plupart des cas, cependant, les déterminés diffèrent de manière beaucoup plus sensible :

Babes' tubercle

Bochdalek's muscle

Bouvereret's ulcer

Brodie's pain

Bruch's glands

Burton's line

Corrigan's sign

Déjerine's bulbar

Doyère's eminence

Durozier's murmur

Galen's anastomosis

Gruber's fossa

Guérin's sinus

Hunter's glossitis

Kirschner's apparatus

Krukenberg's arm, hand

Kussmaul's respiration
Nodule de Babes

Fibres triticéoglosses de Bochdalek

Angine de Bouveret

Névralgie articulaire de Brodie

Plaques de Bruch

Liseré de Burton

Pouls de Corrigan

Syndrome interolivaire de Déjerine

Plaque motrice de Doyère

Double souffle de Durozier

Anse nerveuse de Galien

Cul-de-sac de Gruber

Cul-de-sac de Guérin

Langue de Hunter

Broche de Kirschner

Moignon de Krukenberg

Rythme de Kussmaul 


\author{
Lieutaud's triangle \\ Luschka's crypts \\ Maurer's dots, stippling \\ Merkel's filtrum \\ Miescher's corpuscles \\ Petri dish \\ Pflüger's tubes \\ Pick's vision \\ Purkinje's fibres \\ Quincke's sign \\ Rathke's columns \\ Retzius' space \\ Siakel's method \\ Schleich's anesthetic \\ Theile's canal \\ Traube's phenomenon \\ Vincent's gingivitis \\ Wreden's sign \\ Zinn's ligament \\ Zuckerkandl's body
}

\author{
Trigone de Lieutaud \\ Glandes de Luschka \\ Granulations de Maurer \\ Fossette centrale de Merkel \\ Tubes de Miescher \\ Boite de Pétri \\ Cordons de Pflüger \\ Hallucination visuelle de Pick \\ Réseau de Purkinje \\ Pouls de Quincke \\ Poutrelles de Rathke \\ Cavité de Retzius \\ Cure de Sakel \\ Mélange de Schleich \\ Sinus transverse de Theile \\ Double ton de Traube \\ Angine ulcéro-membraneuse de Vincent \\ Épreuve de Wreden \\ Anneau de Zinn \\ Organe de Zuckerkandl
}

Quelques éponymes anglais construits avec le génitif français présentent la même particularité :

Ossicles of Bertin

Fissure of Bichat

Side fibril of Golgi

Fissure of Rolando

Body of Rosenmüller

Brushes of Ruffini

Recess, space of Tröltsch
Cornets de Bertin

Grande fente de Bichat

Réseau de Golgi

Scissure de Rolando

Organe de Rosenmüller

Corpuscules de Ruffini

Poche antérieure de Tröltsch

\subsubsection{Formes composées}

Comme dans les formes simples, la différence peut ne porter que sur des nuances :

Conradi-Hünermann syndrome

Hallervorden-Spatz syndrome

Hanot-Chauffard syndrome

Posada-Wernicke syndrome
Maladie de Conradi-Hünermann

Maladie de Hallervorden-Spatz

Maladie de Hanot-Chauffard

Maladie de Posada-Wernicke

Mais, souvent, la substitution est plus importante :

\author{
Aran-Duchenne atrophy \\ Arnold-Chiari deformity \\ Charcot-Marie-Tooth atrophy \\ Duchenne-Erb paralysis \\ Kaes-Bechterew layer \\ Landouzy-Déjerine dystrophy \\ Preisz-Nocard bacillus \\ Werding-Hoffmann paralysys \\ Westphal-Piltz phenomenon
}

Amyotrophie d'Aran-Duchenne Syndrome d'Arnold-Chiari Amyotrophie de Charcot-Marie-Tooth Syndrome de Duchenne-Erb Stries de Kaes-Bechterew Atrophie musculaire de Landouzy-Déjerine Corynebacterium de Preisz-Nocard Amyotrophie de Werding-Hoffmann Réflexe de Westphal-Piltz 


\subsection{ADJECTIVATION DE L'ÉPONYME}

L'éponyme peut se trouver adjectivé dans les deux langues ou dans l'une d'elles seulement.

\subsubsection{Adjectivation en anglais et en français}

Basedowified goiter
Brownian movement
Hippocratic face
Jacksonian epilepsy
Rolandic region
Sylvian vein
Vidain plexus
Wallerian degeneration
Wormian bones

Goitre basedowifiant

Mouvement brownien

Faciès hippocratique

Épilepsie Bravais-Jacksonienne, épilepsie bravaisienne

Région rolandique

Veine sylvienne

Rameaux du nerf vidien

Dégénérescence wallérienne

Os wormiens

\subsubsection{Adjectivation en anglais seulement}

\subsubsection{Avec maintien de l'éponyme en français}

$\begin{array}{ll}\text { Darwinian tubercle } & \text { Tubercule de Darwin } \\ \text { Eustachian tube } & \text { Trompe d'Eustache } \\ \text { Fallopian aqueduct } & \text { Aqueduc de Fallope } \\ \text { Gasserian ganglion } & \text { Ganglion de Gasser } \\ \text { Graafian follicle } & \text { Follicule de Graaf } \\ \text { Haversian glands } & \text { Glandes de Havers } \\ \text { Malpighian corpuscles } & \text { Corpuscules de Malpighi } \\ \text { Meibomian glands } & \text { Glandes de Meibomius } \\ \text { Müllerian duct } & \text { Canal de Müller } \\ \text { Pacchionian depression } & \text { Fossette de Pacchioni } \\ \text { Schneiderian membrane } & \text { Membrane de Schneider } \\ \text { Spigelian lobe } & \text { Lobe de Spiegel } \\ \text { Thebesian veins } & \text { Veines de Thébésius } \\ \text { Wolffian body } & \text { Corps de Wolff }\end{array}$

1.6.2.2 Avec disparition de l'éponyme en français

$\begin{array}{ll}\text { Eberthian strumitis } & \text { Thyroïdite typhique } \\ \text { Eustachian catheter } & \text { Sonde tubaire } \\ \text { Eustachian muscle } & \text { Muscle de marteau } \\ \text { Fallopian pregnancy } & \text { Grossesse tubaire } \\ \text { Hunterian chancre } & \text { Chancre induré } \\ \text { Langerhansian adenoma } & \text { Insulome } \\ \text { Meibomian cyst } & \text { Chalazion } \\ \text { Tagliacotian rhynoplasty } & \text { Rhinoplastie à l'italienne } \\ \text { Vesalian bone } & \text { Sésamoïde du jumeau externe } \\ \text { Zeisian sty } & \text { Orgelet marginal }\end{array}$

\subsubsection{Adjectivation en français seulement}

Achilles' reflex

Addison-Biermer anemia
Réflexe achilléen

Anémie biermérienne 


$\begin{array}{ll}\text { Bright's granulations } & \text { Granulations brightiques } \\ \text { Korsakoff's contact potencies } & \text { Dilutions korsakoviennes } \\ \text { Nougaret night blindness } & \text { Héméralopie nougarienne } \\ \text { Paget's disease } & \text { Ostéose pagétique } \\ \text { Parkinson's facies } & \text { Faciès parkinsonien } \\ \text { Pott's paraplegia } & \text { Paraplégie pottique }\end{array}$

Plus rarement, l'éponyme disparaît en anglais :

Pretibial myxedema

Dermopathie basedowienne

Trigeminal impression

Fossette gassérienne

\subsection{SUBSTANTIVATION DE L'ÉPONYME}

\subsubsection{Substantivation en anglais et en français}

Bartholinitis
Brightism
Brucellosis
Buckytherapy
Cowperitis
Curietherapy
D'Arsonvalization
Descemetocele
Douglasitis
Faradization
Leishmaniosis
Meibomitis
Nocardiosis
Parkinsonism
Quenuthoracoplasty
Sichwannoma
Teslaization
Tysonitis

Batholinite
Brightisme
Brucellose
Buckythérapie
Cowpérite
Curiethérapie
D'Arsonvalisation
Descemétocèle
Douglassite
Faradisation
Leishmaniose
Meibomite
Nocardiose
Parkinsonisme
Quénuthoracoplastie
Schwannome
D'Arsonvalisation
Tysonite

Parfois, l'éponyme substantivé est doublé en anglais d'une forme adjectivée ou d'un génitif :
Bartholinian abscess
Bartholinite

B'right's disease

Brightisme

\subsubsection{Substantivation en anglais seulement}

Esmarch
Franklinization
Heurteloup
Kupferoma
Folitzerization
Wucheriasis

Bande d'Esmarch

Bain statique

Sangsue artificielle de Heurteloup

Endothéliome kupfférien

Méthode de Politzer

Filariose à Wucheria

\subsubsection{Substantivation en français seulement}

Basedowiform disease

Béniqué's sound
Basedowisme

Béniqué 
Il arrive même que l'éponyme disparaisse en anglais :

Radium implantation

Curiepuncture

\section{2. ÉPONYMES DIFFÉRENTS DANS LES DEUX LANGUES}

Dans un grand nombre de cas, les éponymes anglais et français ne se superposent pas. Les différences qui en résultent peuvent prendre des formes très variées.

\subsection{L'ÉPONYME ANGLAIS EST ENCORE PRÉSENT EN FRANÇAIS}

\subsubsection{En association avec un autre éponyme}

Bergmann's cells

Bruhl's disease

Caffey's disease

Colles' fracture

Doléris' operation

Dressler's disease

Dühring's disease

Fahr-Volhard disease

Fröhlich's disease

Gilbert's disease

Graham-Little syndrome

Gruby's disease

Hürthle cell tumor

Köhler's disease

Krabbe's disease

Lederer's anemia

Nonne's syndrome

Shiga's bacillus

Still's disease

Türck's bundle

Turner's syndrome

Unverricht's disease

Van Neck's disease

Weil's disease

Werner's syndrome

Winckel's disease
Cellules de Golgi-Bergmann

Maladie de Debove-Bruhl

Syndrome de Caffey-Smith

Fracture de Pouteau-Colles

Opération de beck-Doléris

Maladie de Dressler-Harley

Maladie de Dühring-Brocq

Maladie de Keith-Fahr-Volhard

Syndrome de Babinski-Fröhlich

Syndrome de Gilbert-Lereboullet

Syndrome de Lassueur-Graham-Little

Maladie de Gruby-Sabouraud

Tumeur de Baber-Hürthle

Maladie de Köhler-Stieda

Syndrome de Christensen-Krabbe

Maladie de Lederer-Brill

Syndrome de Nonne-Marie

Bacille de Shiga-Kruse

Maladie de Chauffard-Still

Faisceau de Türck-Meynert

Syndrome de Turner-Albright

Syndrome d'Unverricht-Lundborg

Maladie de Van Neck-Odelberg

Maladie de Mathieu-Weil

Syndrome de Werner-Rothmund

Maladie de Charrin-Winckel

\subsubsection{Par réduction d'une telle association}

Aberhalden-Fanconi syndrome

Baldy-Webster operation

Clérambault-Kandinsky complex

Ehrlich-Heinz granules

Itard-Cholewa sign

Laurence-Moon-Biedl syndrome

Lhermitte-McAlpine syndrome

Monro-Richter line

Ollier-Thiersch graft

Putnam-Dana syndrome
Syndrome de Fanconi

Opération de Baldy

Syndrome de Clérambault

Corpuscules de Heinz

Signe d'Itard

Syndrome de Laurence-Biedl

Syndrome de Lhermitte

Ligne de Monro

Greffe de Thiersch

Maladie de Putnam 
Quénu-Mayo operation Sucquet-Hoyer canals

Taussig-Bing syndrome
Opération de Quénu

Canaux de Sucquet

Syndrome de Taussig

\subsubsection{En combinaison variable avec d'autres éponymes}

$\begin{array}{ll}\begin{array}{l}\text { Aberhalden-Franconi syndrome, Lignac-Fantoni } \\ \text { syndrome }\end{array} & \text { Syndrome de Toni-Franconi } \\ \begin{array}{l}\text { Bloch-Sulzberger disease } \\ \text { Calvé-Perther disease, Legg-Calvé disease }\end{array} & \text { Dermatose pigmentaire de Siemens-Bloch } \\ & \begin{array}{c}\text { Maladie de Perthes-Calvé-Legg, maladie de } \\ \text { Waldenström-Perthes }\end{array} \\ \text { Fede's disease, Riga-Fede disease } & \text { Maladie de Cardarelli-Fede-Riga } \\ \text { Larsen's disease, Larsen-Johansson disease } & \text { Maladie de Sven-Johansson-Sindig-Larsen } \\ \text { Lichtheim's disease, } & \text { Syndrome de Lichtheim-Dana }\end{array}$

Putnam-Dana syndrome

Syndrome de Lichtheim-Dana

\subsection{L'ÉPONYME ANGLAIS EST SUBSTITUÉ EN FRANÇAIS}

\section{Auspitz's dermatosis \\ Babcock's operation \\ Bateman's disease \\ Bigelow's septum}

Bruce's bundle

Brugsch's disease, Timme's syndrome

Carman's sign

Cavaré's disease

Deiters' phalangeal cells

Devergie's disease

Isthmus of the Fallopian tubes

$J$. Fothergill's disease

Guérin-Stern syndrome

His" canal

Kussmaul's aphasia

Michon's eosinophilic granuloma

Morgagnian caruncle

Pel-Ebstein pyrexia

Pott's asthma

Retzius' fibres

Rosenmüller's gland

Rassbach's disease

Rovighi's sign

Rust's disease

Schüller's glands

Siemens' syndrome

Smith's fracture, reverse Colles' fracture

Siühmer's disease

Wagstaffe's fracture
Maladie d'Alibert-Bazin

Opération de Fredet

Acné varioliforme de Bazin, epithelioma contagiosum de Neisser

Éperon de Merkel, lame osseuse soustrochantérienne de Rodet

Zone de Westphal

Microsomie de Pahon

Signe du ménisque de Guéret et Lambling

Maladie de Westphal

Cellules de soutien de l'organe de Corti

Lichen rubert acuminatus Kaposi

Isthme de Barkow

Angine d'Henoch

Poupées de bois de Rocher

Canal de Bochdalek

Syndrome de Worster-Draught-Allen

Granulome histiocytaire de Schajowicz et Polak

Barrière de Mercier

Forme pyrétique à rechute de la maladie de Hodgkin

Asthme thymique de Koop

Filaments des cellules de Deiters

Ganglion de Cloquet

Gastroxie de Lépine

Signe de Recamier

Mal de Pott sous-occipital

Diverticules du canal de Gartner

Syndrome de Jacquet

Fracture de Goyrand

Balanite profonde interstitielle de Tournier

Fracture malléolaire externe de Lefort 


\section{3 ÉPONYMES SUPPLÉMENTAIRES}

Il n'est pas rare de voir une notion anatomique, physiologique, pathologique ou autre désignée par plusieurs éponymes, lesquels ne se superposent pas nécessairement dans les deux langues. L'anglais surtout recourt souvent à cette synonymie éponymique.

\subsection{1 Éponymes supplémentaires en anglais}

Achalme's bacillus/Welch's bacillus

Amici's disk/Dobie's line, Krausse's membrane Aran's green cancer/Balfour's disease

Aran-Duchenne atrophy/Cruveilheir's paralysis

Baelz's disease/Puente's disease

Bandl's ring/Braun's ring

Basedow's disease/Flajani's disease, Graves' disease, Parry's disease

Bauhin's valve/valve of Tulpius, valve of Varolius Beigl's disease/Chignon's disease

Bence-Jones albumosuria/Bradshaw's albumosuria Benedikt's syndrome/Claude's syndrome

Bertin's bone/Wistar's pyramid

Bertin's ligament/Bigelow's ligament

Bjork's syndrome/Cassidy-Scholte syndrome

Bouillaud's disease/Beauvais' disease

Bürger's sign/Carel's sign

Cazenave's lupus/Biette's lupus

Charcot-Leyden crystals/Leyden's crystals, Zenker's crystals

Clarke's column/Gierke's respiratory bundle. Krause's bundle, Stilling's column

Cloquet's ligament/Haller's habenula

Darier's disease/White's disease

Deiter's nucleus/Laurer's nucleus

Denonvilliers' aponeurosis/ Tyrrell's fascia

Dittrich's plugs/Traube's plugs

Douglas' fold/Mackenrodt's ligament

Dresbach's anemia/Herrick's anemia

Drysdale's corpuscles/Bennett's small corpuscles

Dupuytren's fracture/Pott's fracture

Hiatus Fallopii/Ferrein's foramen, Tarin's foramen

Fauchard's disease/Magitot's disease, Rigg's disease

Flechsig's oval bundle/Bruce's tract

Flexner's bacillus/Strong's bacillus, HissandRussell Y bacillus

Gianuzzi's crescents/demilunes of Heidenhain

Grancher's disease/Desnos' disease

Grandy's corpuscles/Merkel's corpuscles

Gratiolet's bundle/Wernicke's fibres

Hanot's disease/Todd's cirrhosis

Hassal's corpuscle/Gierke's corpuscle, Leber's corpuscle

Heister's valve/Anussat's valve
Bacille d'Achalme

Strie d'Amici

Cancer vert d'Aran

Amyotrophie d'Aran-Duchenne

Maladie de Baelz

Anneau de Bandl

Maladie de Basedow

Valvule de Bauhin

Maladie de Beigl

Albumosorie de Bence-Jones

Syndrome de Benedikt

Cornet de Bertin

Ligament de Bertin

Syndrome de Bjork

Maladie de Bouillaud

Signe de Bürger

Lupus de Cazenave

Cristaux de Charcot-Leyden

Colonne grêle de Clarke

Ligament de Cloquet

Maladie de Darier

Noyau de Deiters

Aponévrose de Denonvilliers

Bouchons de Dittrich

Repli de Douglas

Syndrome de Dresbach

Corpuscules de Drysdale

Fracture de Dupuytren

Hiatus de Fallope

Maladie de Fauchard

Faisceau de Flechsig

Bacille de Flexner

Croissants de Gianuzzi

Maladie de Grancher

Corpuscules de Grandy

Radiation optique de Gratiolet

Maladie de Hanot

Corpuscule de Hassal

Valvule de Fieister 
Sheath of Henle/Ruffini's subsidiary sheath of Key and Retzius

Hensen's canal/Reichert's canal

Bundle of His/Kent-His bundle, bundle of StanleyHis, Gaskell's bridge

Hoffmann's sign/Mayer's reflex

Kahler's disease/Bozzolo's disease, Huppert's disease

Köhler second disease/Freiberg-Köhler disease, Freiberg's infarction, Panner's disease

Koplik's sign/Comby's sign, Filatow's spots, Flindt's spots

Laumonier's ganglion/Bock's ganglion

Leydig's cells/Gley's cells

Lieberkiihn's glands/Galeioti's glands

Lissauer's marginal zone/Spitzka's bundle, column of Spitzka-Lissauer

Marie's ataxia/Sanger-Brown ataxia

Meige's disease/Milroy's disease, Nonne-Milroy Meige syndrome

Meissner's plexus/Remak's plexus

Naboth's ovules/Montgomery's follicles

Nunn's gorges corpuscles/Bennett's large corpuscles

Lines of Owen/Salter's lines, Schreger's lines

Paneth's cell/Davidoff's cell

Pirogoff's triangle/Pinaud's triangle

Plummer-Vinson syndrome/Paterson-Kelly syndrome

Quincke's edema/Bannister's disease, Milton's edema

Reclus' disease/Copper's disease

Reil's sulcus/Burdach's fissure

Riga's aphthae/Cardarelli's aphthae

R.okitansky's disease/Budd's jaundice

Rose's tetanus/Janin's tetanus, Klemm's tetanus

Sheehan's syndrome/Chiari-Frommel disease,

Frommel's disease

Skene's glands/Guérin's glands

Sonne's bacillus/Duval's bacillus

Spiegler-Fendt sarcoid/Bäfverstedt's disease

Stahli's pigment line/Hudson's line

Sternberg's giant cells/Sternberg-Reed cells, Reed's cells, Hodgkin's cells

Tenon's capsule/Bonnet's capsule

Tournay's sign/Gianelli's sign

Troisier's ganglion/Virchow's node

Foramen of Vicq d'Azyr/Schwalbe's foramen

Valve of Vieussens/Willis' valve

Von Hippel's disease/Lindau's disease, Lindau-von Hippel disease

Wolffian body/Oken's body

Wormian bones/Andernach's ossicles
Gaine de Henle

Canal de Hensen

Faisceau de His

Signe de Hoffmann

Maladie de Kahler

Deuxième maladie de Köhler

Signe de Koplik

Ganglion de Laumonier

Cellules de Leydig

Glandes de Lieberkühn

Zone marginale de Lissauer

Hérédo-ataxie cérébelleuse de Marie

Maladie de Meige

Plexus de Meissner

CEufs de Naboth

Corpuscules de Nunn

Lignes de Owen

Cellule de Paneth

Triangle de Pirogoff

Syndrome de Plummer-Vinson

OEdème de Quincke

Maladie de Reclus

Sillon de Reil

Aphtes de Riga

Maladie de Rokitansky

Tétanos céphalique de Rose

Syndrome de Sheehan

Glandes de Skene

Bacille de Sonne

Sarcoïde de Spiegler-Fendt

Ligne de Stahli

Cellules de Sternberg

Capsule de Tenon

Phénomène de Tournay

Ganglion de Troisier

Trou borgne de Vicq d'Azyr

Valvule de Vieussens

Maladie de von Hippel

Corps de Wolff

Os wormiens 

vantage :

Même lorsque le français possède plusieurs éponymes, l'anglais en offre encore da-

Bartholin's gland, Duverney's gland/ Tiedemann's gland

Bayle's disease, Vogt-Spielmeyer disease/BettenMayou disease

Demour's membrane, Descemet's membrane/ Duddell's membrane

Hurler's disease, Pfaudler-Hurler disease/HunterHurler disease

Huschke's valve, Rosenmüller's valve/ Hasner's valve
Glande de Bartholin, glande de Joseph de Verney

Maladie de Bayle, maladie de Vogt-Spielmeyer

Membrane de Demours, membrane de Descemet

Polydistrophie de Hurler, syndrome de PfaundlerHurler

Valvule de Huschke, valvule de Rosenmüller

\subsection{2 Éponymes supplémentaires en français}

Brïcke muscle

Déjerine's bulbar syndrome

Déjerine's parietal syndrome

Di Guglielmo's disease

Economo's disease

Erichsen's disease

Henderson-Jones disease

Jacobson's cartilage

Kaposi's disease

Kultschitzky's cells

Libman-Sacks disease

Lobstein's disease

Morschowitz's disease

Olmer's disease, Conor and Bruch disease

Preiser's disease

Rouget's muscle

Spitzka's nucleus

Stainton's disease

Steir-Leventhal syndrome

Urisberg's cartilage
Muscle de Brücke/muscle de Wallace

Syndrome interolivaire de Déjerine/syndrome paramédian de Foix

Syndrome de Déjerine-Verger/topoanesthésie de Claude

Maladie de Di Guglielmo/érythromyélose de Chevalier

Maladie de von Economo/maladie de Cruchet

Maladie d'Erichsen/syndrome de Souques

Maladie de Henderson-Jones/maladie de Reichel

Cartilage de Jacobson/maladie de Huschke

Xeroderma pigmentosum de Kaposi/maladie pigmentaire épithéliomateuse de Quinquaud, mélanose lenticulaire progressive de Pick

Cellules de Kultschitzky/cellules de Nicolas

Maladie de Libman-Sacks/syndrome de KaposiBesner-Libman-Sacks

Maladie de Lobstein/syndrome de Van Hoewe

Maladie de Morschowitz/anémie hémolytique micro-angiopathique thrombotique de Symmer

Maladie d'Olmer, maladie de Conor et Bruch/ fièvre boutonneuse de Carducci

Maladie de Preiser/maladie de Koehler-Mouchet

Muscle de Rouget/fibres de Müller

Noyau de Spitzka/noyau de Siemerling

Syndrome de Stainton/maladie de CapdepontHodge, dentine opalescente héréditaire de Hodge

Maladie de Stein-Leventhal/maladie de Marnou

Cartilage de Wrisberg/cartilage de Morgagni

\subsection{3 Éponymes supplémentaires dans les deux langues}

Adie's syndrome/Holmes-Adie syndrome

Béraud's valve/Arnold's fold

Besnier-Boeck-Schaumann disease, sarcoid of Boeck/Chaumann's disease

Gee's disease/Gee-Herter disease, Gee-Herter-

Heubner disease, Herter-Heubner disease, Gee-

Thaysen disease
Maladie d'Adie/syndrome de Weill et Reys Valvule de Béraud/valvule de Krause

Maladie de Besnier-Boeck-Schaumann, sarcoïde de Boeck/lupus pernio de Besnier-Tenneson

Maladie de Gee/maladie de Herter 
Hodgkin's disease, Sternberg's disease/Billroth's disease, Ebstein-Pel disease MurchinsonLanderson syndrome

Lorain's infantilism/Leroy-Lorain infantilism, Paltauf's dwarfism

Santorini's canal/Bernard's canal

Schlatter-Osgood disease/Schlatter's disease

Van der Hoeve's syndrome, Adair-Dighton syndrome/Eddowes' syndrome, SpumayEddowes syndrome

Vrolik's disease/Durante-Porak disease

Lichen albus of Zumbusch/Osillag's disease
Maladie de Hodgkin, maladie de Sternberg/ maladie de Paltauf

Infantilisme de Lorain/chétivisme de Bauer, juvénilisme d'Apert

Canal de Santorini/canal accessoire de Wirsung

Maladie de Schlatter-Osgood/maladie de Lannelongue

Syndrome de Van der Hoeve, syndrome d'Adair-

Dighton/maladie d'Apert, syndrome de Lobstein-Carnazon

Maladie de Vrolik/osteogensis congenita de Looser

Lichen albus de Zumbusch/scléro-lichen de Gougerot

\subsection{INVERSION D'ÉPONYMES}

En passant d'une langue à l'autre, il arrive que les éponymes changent de place dans l'association qui les réunit.

\subsubsection{L'inversion éponymique existe à côté de l'ordre initial en anglais}

Bucky-Potter disease, Potter-Bucky disease

Christian-Weber disease, Weber-Christian disease

Horner-Bernard syndrome, Bernard-Horner syndrome

Murray-Stout syndrome, Stout-Murray syndrome

Tract of Philippe-Gombault, Gombault-Philippe triangle

Stevens-Johnson syndrome, Johnson-Stevens syndrome

Sill-Chauffard syndrome, Chauffard-Still syndrome

Wymburn-Mason syndrome, Mason-Wynburn syndrome
Maladie de Potter-Bucky

Maladie de Weber-Christian

Syndrome de Bernard-Horner

Syndrome de Stout-Murray

Faisceau de Gombault et Philippe

Syndrome de Johnson-Stevens

Syndrome de Chauffard-Still

Syndrome de Mason-Wynburn

\subsubsection{L'inversion éponymique n'existe pas en anglais}

Adams-Stokes syndrome

Bamberger-Marie disease

Erb-Charcot disease

Filatow-Dukes disease

Flateau-Schilder disease

Holmes-Stewart phenomenon

Jakob-Creutzfeldt disease

Mendel-Bechterew reflex

Osler-Rendu disease

Phocas-Tillaux disease

Strümpell-Westphal pseudosclerosis
Syndrome de Stokes-Adams

Maladie de Pierre Marie-Bamberger

Maladie de Charcot-Erb

Maladie de Dukes-Filatow

Maladie de Schilder-Flateau

Phénomène de Stewart-Holmes

Maladie de Creutzfeldt-Jakob

Réflexe de Bechterew-Mendel

Maladie de Rendu-Osler

Maladie de Tillaux-Phocas

Syndrome de Westphal-Strümpell 


\section{ABSENCE D'ÉPONYME DANS L'UNE OU L'AUTRE LANGUE}

\subsection{L'ÉPONYME N'EXISTE QU'EN ANGLAIS}

\subsubsection{Formes simples}

3.1.1.1 Maintien intégral du déterminé et suppression du déterminant éponymique en français

Budge's cilio-spinal centre

Centre cilio-spinal

Deiters' neuroglia cells

Hunt's striatal syndrome

Cellules névrogliques

Sappey's subareolar plexus

Syndrome strié

Sergent's white adrenal line

Plexus sous-aréolaire

Verga's lacrymal groove

Ligne blanche surrénale

Gouttière lacrymale

Parfois, le déterminé conservé est étoffé d'une précision supplémentaire :

Caspar's ring opacity

Opacité annulaire de la cornée

Langerhans' wandering cells

Cellules migratrices de la cornée

\subsubsection{Maintien du déterminé et substitution du déterminant éponymique en} français

Barclay's niche

Billroth's hypertrophy

Birkett's hernia

Boerhaave's glands

Botella's ligament

Bretonneau's angina

Brodie's ligament

Buchwald's atrophy

Bunge's amputation

Charcot's fever

Cloquet's septum

Cobelli's glands

Cruveilhier's joint

Czerny's diathesis

Deventer's pelvis

Dimmer's keratitis

Dragstedt's graft

Duchenne's paralysis

Dupuytren's phlegmon

Ebstein's leukemia

Edelman's anemia

Ferrein's tubule

Fiedler's myocarditis

Gilbert's cholemia

Gombault's neuritis

Gower's contraction

Haller's cones

Hander's pelvis

Henle's ampulla
Niche duodénale

Hypertrophie du pylore

Hernie synoviale

Glandes sudoripares

Ligament artériel

Angine diphtérique

Ligament huméral transverse

Atrophie cutanée progressive

Amputation périostée

Fièvre biliaire intermittent

Septum crural

Glandes cesophagiennes

Articulation atloïdo-odontoïdienne

Diathèse exsudative

Bassin plat

Kératite en grillage

Greffe en accordéon

Paralysie bulbaire

Phlegmon du cou

Leucémie aiguë

Anémie infectieuse chronique

Tubule urinifère contourné

Myocardite aiguë

Cholémie familiale

Névrite périaxile

Contraction des solaires

Cônes afférents

Bassin épineux

Ampoule du cana déférent 


Hilliard's lupus
Hilton's recess
Holthouse's hernia
Horton's headache
Hunter's ligament
Hutchinson's prurigo
Jacobson's plexus
Jenner's emphysema
Kilian's pelvis
Koyter's muscle
Kronecker's centre
Kussmaul's coma
Lane's kink
Liepmann's apraxia
Lockwood's ligament
Ludwig's labyrinth
Mallory's leukemia
Menson's schistosomiasis
Monro's bursa
Fatterson's corpuscles
Folitzer's cone
Pott's gangrene
Furkinje's vesicle
Quain's heart
Quinzke's meningitis
Reichel's chondromatosis
Richet's aneurysm
Riesman's myocardosis
Rokitansky's pelvis
Rollet's stroma
Sampson's cyst
Santorini's muscle
Schmorl's jaundice
Sichrötter's chorea
Shekelton's aneurysm
Stilling's fibres
Sydenham's cough
Turner's sulcus
Wassmann's glands
Well's facies
Wrisberg's ligament
Zuckerkandl's convolution

Lupus marginé

Logette des osselets

Hernie inguino-crurale

Céphalée histaminique

Ligament rond de l'utérus

Prurigo estival

Plexus tympanique

Emphysème sénile

Bassin ostéo-malacique

Muscle sourcilier

Centre cardio-inhibiteur

Coma diabétique

Coudure intestinale

Apraxie motrice

Ligament suspenseur du globe oculaire

Labyrinthe rénal

Leucémie benzolique

Schistosomiase intestinale

Bourse oléocrânienne

Corpuscules du molluscum

Cône lumineux

Gangrène sénile

Vésicule germinative

Cour gras

Méningite séreuse

Chondromatose articulatoire

Anévrisme fusiforme

Myocardose dégénérative

Bassin spondylolisthésique

Stroma des globules rouges

Kyste goudronneux

Muscle risorius

Ictère nucléaire

Chorée laryngée

Anévrisme disséquant

Fibres d'association du cervelet

Toux hystérique

Sillon pariétal

Glandes peptiques

Faciès ovarien

Ligament du ménisque externe

Circonvolution sous-calleuse

\subsubsection{Transformation de tous les éléments en français}

3.1.1.3.1 Par simple explicitation du déterminé disease, syndrome, operation, etc.

\author{
Ballingall's disease \\ Bateman's disease \\ Blum's syndrome \\ Breisky's disease
}

Mycétome

Purpura sénile

Azotémie chloropénique

Kraurosis vulvae 
Chester's disease

Dalrymple's disease

Dutton's disease

Eale's disease

Fenwick's disease

Fowler's operation

Gibney's disease

Grawitz's degeneration

Hall's disease

Harries' syndrome

Hirschfeld's disease

Holmer's degeneration

Hyde's disease

Janet's disease

König's syndrome

Lane's disease

Loriga's disease

Marlin's syndrome

Mill's disease

Miller's disease

Moore's syndrome

Mortimer's disease

Munk's disease

Neumann's disease

Ogilvie's syndrome

o'Higgins' disease

Parke's syndrome

Petit's disease

Pottain's disease

Rayer's disease

Rowland's disease

Saville's disease

Selye's syndrome

Thomson's disease

Vidal's disease

Watson's disease

Wernicke's syndrome

Whitmore's disease

Whytt's disease

Wilkins' syndrome

3.1.1.3.2 Par un procédé plus élaboré

Alligham's ulcer

Altmann's granule

Arroyo's sign

Baker's cyst

Berger's rhythm

Bright's blindness

Broadbent's apoplexia

Brunn's membrane
Xanthomatose des os longs

Cyclokératite

Trypanosomiase

Périphlébite rétinienne

Atrophie primitive de l'estomac

Pleurectomie

Périspondylite

Basophilie

Pseudohydrocéphale

Hypoglycémie spontanée

Diabète aigu

Atrophie cérébelleuse

Lichénification nodulaire

Psychasténie

Ostéochondrite disséquante

Stase intestinale chronique

Doigt mort

Anémie phagocytaire

Hépiplégie spinale ascendante

Ostéomalacie

Epilepsie abdominale

Lupus vulgaire non ulcéré

Néphrose lipoïdique

Pemphigus végétant

Iléus spasmodique

Néphroso-néphrite hémorragique

Vomissements acétoniques de l'enfance

Hernie diaphragmatique

CEdème pulmonaire

Xanthome

Xanthomatose

Dermatite exfoliative épidémique

Syndrome général d'adaptation

Poîkilodermose congénitale

Névrodermite

Porphyrie abdominale

Démence sénile

Mélioïdose

Hydrocéphalie interne

Hyperplasie congénitale des surrénales

Fissure anale

Mitochondrie

Paresse papillaire

Bursite

Ondes alpha

Amaurose urémique

Inondation ventriculaire

Epithélium olfactif 
Camper's ligament

Charcot's arthritis

Cooper's irritable breast

Corlett's pyosis

Cowper's ligament

Day's factor

Donne's bodies

Fielding's membrane

Galen's pore

Gaul's pits

Gower's intermediate process

Gull's renal epistaxis

Haller's tripod

Henoch's chorea

Hunter's gubernaculum

Jackson's sign

Klieg's eye

Kölliker's nucleus

Lecat's gulf

Leyden's ataxia

Lucas' sign

Luschka's cartilage

Madelung's neck

McCall's festoon

Mickulicz's aphthae

Montgomery's cups

Morgagni's tubercle

Morrison's pouch

Müller's dust bodies

Parrot's atrophia

Rauber's layer

Ray's mania

Rokitansky's kidney

Sappey's ligament

Sander's sign

Scarpa's membrane

Steida's process

Symington's body

Traube s murmur

Unna's dermatitis

Wagner's spot

Weber's organ

Wernicke's triangle

Westphal's contraction

Willan's lepra

Willis' pouch

Winslow's stars
Aponévrose périnéale profonde

Arthropathie tabétique

Mastodynie

Impétigo bulleux

Insertion pubienne du fascia lata

Acide folique

Globules du colostrum

Tapétum

Canal inguinal

Érosions de la cornée

Corne latérale de la moelle

Hématurie rénale essentielle

Trone coeliaque

Tic spasmodique

Ligament du testicule

Respiration asthmatique

Conjonctivite des cinéastes

Substance gélatineuse centrale

Cul-de-sac bulbaire

Pseudotabès

Gros ventre rachitique

Nodule glottique antérieur

Lipomatose cervicale

Tuméfaction du feston gingival

Stomatite aphteuse récidivante

Glandes utérines

Bulbe olfactif

Repli hépato-rénal

Hémoconies

Athrepsie

Blastoderme

Perversion instinctive

Amyloïdisme rénal

Frein méniscal postérieur

Syncinésie bouche-main

Tympan secondaire

Tubercule externe de l'astragale

Raphé du coccygien

Bruit de galop

Eczéma séborrhéique

Plaque germinative

Utricule prostatique

Segment lenticulo-optique

Phénomène paradoxal du pied

Psoriasis

Epiploon gastro-hépatique

Tourbillon veineux

\subsubsection{Multiplication de l'éponyme en anglais}

Abercrombie's degeneration, Virchow's degeneration Dégénérescence amyloïde 
Abrami's disease, Widal-Abrami disease Albert's keloid, Hawkins' keloid

Appolito's suture, Gely's suture Bamberger's disease, Gower's disease Blessig's cysts, Iwanoff's retinal edema Bowman's muscle, Crapton's muscle Bozzi's foramen, Soemmering's spot Breda's disease, Charlouis' disease Bright's murmur, Beatty-Bright friction mumur Bruton's syndrome, MacQuarrie's syndrome Campbell's ligament, Gerdy's ligament Carrion's disease, Fonseca's disease, Pedroso's disease

Clapton's line, Corrigan's line Corning's puncture, Quincke's puncture Corrigan's pneumonia, Kaufmann's pneumonia Elsner's asthma, Heberden's asthma Engelmann's disk, Hensen's disk Francis' disease, Ohara's disease Held's bundle, Marchi's bundle, Löwenthal's tract Holth's operation, Elliot's operation Hutinel's erythema, Stricker's disease Ionnesco's fossa, fossa of Treitz Kiesselbach's area, Little's area Leriche's compression, Naffziger's syndrome Pastia's sign. Thomson's sign Rouget's cell, Marchand's cell Schlesinger's sign, Pool's phenomenon Toynbee's corpuscle, Virchow's corpuscle Willis' pancreas, Winslow's pancreas
Ictère hémolytique acquis

Chéloïde cicatricielle

Suture intestinale continue

Chorée saltatoire

Dégénérescence kystique de la rétine

Muscle ciliaire

Tache jaune

Pian

Frottement pleural

Agammaglobulinémie

Ligament suspenseur de l'aisselle

Chromoblastomycose

Liseré cuivrique

Ponction lombaire

Pneumonie interstitielle aiguë

Angine de poitrine

Mésophragme

Tularémie

Faisceau tecto-spinal

Sclérectomie

Erythème infectieux aigu

Fossette mésocolique

Tache vasculaire

Syndrome du scalène

Stries transversales du pli du coude

Cellule adventitielle

Phénomène des jambes

Cellule fixe de la cornée

Pancréas accessoire

3.1.1.5 Plus exceptionnellement, l'absence d'éponyme en français s'observe après une forme anglaise adjectivée ou construite avec le génitif français

Langerhansian hormone

Sigaultian section

Stigmata of Benecki

Foramen of Bochdalek

Glia of Fanana

Interfibrillar substance of Flemming

Glands of Gay

Bundle of Helweg

Triad of Hertz

Gyrus of Heschl

Muscle of Jung

Pores of Kohn

Columns of Kölliker

Vestigial fold of Marshall

Duct of Pecquet

Gyrus of Andreas Retzius

White substance of Schwann

Band of Tarinus
Insuline

Symphysectomie

Ulcérations multiple de la grande courbure

Hiatus pluero-péritonéal

Névroglie de l'écorce cérébelleuse

Hyaloplasme

Glandes péri-anales

Faisceau olivaire de la moelle cervicale

Cardiasthénie

Circonvolution temporale transverse

Muscle pyramidal de l'oreille

Pores des alvéoles

Myofibrilles

Pli vestigial du péricarde

Canal thoracique

Circonvolution du corps calleux

Myéline

Bandelette semi-circulaire 


\subsubsection{Formes composées}

3.1.2.1 Maintien du déterminé et substitution du déterminant éponymique en français

Charcot-Neumann crystals

Cristaux du sperme

Cheyne-Stokes asthma

Asthme cardiaque

Epper-Cesa-Bianchi cirrhosis

Cirrhose réticulo-endothéliale

Liddell and Sherrington reflex

Réflexe myostatique

Marshall-Hall facies

Faciès hydrocéphalique

Obersteiner-Redlich area

Aire opaque

Prowazek-Greeff bodies

Corpuscules du trachome

Sirümpell-Leichtenstern encephalitis

Encéphalite aiguee hémorragique primitive

\subsubsection{Transformation de tous les éléments en français}

3.1.2.2.1 Par simple explicitation du déterminé disease, syndrome, etc.

Albright-Butler-Bloombey syndrome

Brown-Symmers disease

Büdinger-Ludloff-Laewen disease

Fuchs-Terrien disease

Hench-Rosenberg syndrome

His-Werner disease

F'ugnier-Jersild syndrome

Hitchinson-Gilford disease

Koenig-Wichman disease

Langdon-Down disease

Letterer-Siwe disease

Lewandowsky-Lutz disease

Lucas-Champonnière's disease

McLean-Maxwell disease

Pierret-Rougier syndrome

Shaver-Riddel disease

Skevas-Zerfus disease

Spielmeyer-Stock disease

\subsection{Par un procédé plus élaboré}

Allen-Doisy hormone

Dohle-Heller aorta

Durand-Reynolds permeability factor

Fazio-Londe atrophy

Ghon-Sachs bacillus

Hernig-Lommel sign

Hübener-Thompson phenomenon

Merkel-Ranvier cells

Neisser-Wechsberg phenomenon

Sneddon-Wilkinson dermatosis
Diabète phosphatique

Encéphalite aiguë séreuse de l'enfance

Fracture du cartilage rotulien

Dégénérescence marginale de la cornée

Rhumatisme palindromique

Fièvre de cinq jours

Éléphantiasis génito-ano-rectal

Nanisme sénile

Pemphigus

Imbécillité mongolienne

Histiocytomatose

Épidermodysplasie verruciforme

Bronchite pseudo-membraneuse chronique

Ostéitite postérieure du calcanéum

Psychose tabétique

Aluminose pulmonaire

Maladie des pêcheurs de perles

Atrophie rétinienne de l'idiotie amaurotique
Amniotine
Aortite syphilitique
Hyaluronidase
Paralysie bulbaire progressive infantile
Vibrion septique
Arythmie respiratoire
Bactério-agglutination
Mélanoblastes cutanés
Déviation du complément
Pustule sous-cornéenne 


\section{L'ÉPONYME N'EXISTE QU'EN FRANÇAIS}

\subsubsection{Formes simples}

3.2.1.1 Maintien du déterminé et suppression du déterminant éponymique en anglais

Plexus tonsillaire d'Anderson

Cellules en panier de Boll

Bandelette diagonale de Broca

Pli polaire de Broca

Noyau semi-lunaire de Flechsig

Phlébite bleue de Grégoire

Percussion paradoxale de Hertz

Bassin fendu de Littman

Faisceau sulco-marginal de Marie

Phlegmon ligneux de Reclus

Nerf nasopalatin de Scarpa

Noyau blanc de Stilling

Cataracte coronaire de Vogt

Nerf accessoire de Willis

Tonsillar plexus

Basket cells

Diagonal band

Polar sulcus

Semilunar nucleus

Blue phlebitis

Paradoxical percussion

Split pelvis

Sulcomarginal tract

Woody phlegmon

Nasopalatine nerve

White nucleus

Coronary cataract

Accessory nerve

Parfois, le déterminé conservé se présente sous la forme latine :

Pli marginal supérieur de Gratiolet

Gyrus supra-marginalis

Lymphodermie maligne de Kaposi

Lymphodermia perniciosa

Substance innominée de Reichert

Substantia innominata

3.2.1.2 Maintien du déterminé et substitution du déterminant éponymique en anglais

Ganglion d'Arnold

Infantilisme de Gandy

Épine de Henle

Ganglion de Hirschfeld

Laryngoscopie de Killian

Granulations de Langley

Triangle de Lecène

Tache de Mariotte

Réflexe de Mayer

Cellule de Schultze

Canal de Stenson

Noyau de Stilling

Cour de Traube

Otic ganglion

Regressive infantilism

Suprameatal spine

Posterior renal ganglion

Suspension laryngoscopy

Cytoplasmic granules

Superior carotid triangle

Blind spot

Finger-thumb reflex

Olfactory cell

Nasopalatine canal

Tegmental nucleus

Ox heart

Dystocie de White

Contracting dystocia

3.2.1.3 Transformation de tous les éléments en anglais

3.2.1.3.1 Par explicitation du déterminé maladie, syndrome, opération, etc.

Maladie de Calvé

Opération de Delorme

Opération de Freund
Platyspondylia

Decortication of lung

Chondrotomy 
Opération de Freyer

Maladie de König

Syndrome de MacLennen

Opération de Matas

Opération de Meredith

Opération d'O'Shaughnessy

\subsection{Par un procédé plus élaboré}

Nerf récurrent d'Arnold

Zone grillagée d'Arnold

Rhumatisme de Bougainville

Pronation douloureuse des jeunes enfants de Broca

Prurigo simplex aigu de Brocq

Liseré de Burton

Pilier interne de l'organe de Corti

Fracture marginale postérieure de Destot

Fossette du ganglion de Gasser

Plaque initiale du pityriasis rosé de Gilbert

Tronc du faisceau de His

Cellule de la tumeur de Krukenberg

Signe de Lasègue

Corps muqueux de Malpighi

Ulcère chronique sous-miné de Meleney

Fossette de Mohrenhiem

Stéatome de Müller

Noyau gustatif de Nageotte

Travées de Remak

Nodule de Schmorl

Funiculite vertébrale de Sicard

Rides de l'aqueduc de Sylvius

Anse oméga de Treves

Septicémie veineuse subaiguë de Vaquez

Couche grise de la valvule de Vieussens

Cercle de Weber

Éperon de Wolff

Membrane de Zeissel
Suprapubic transvesical prostatectomy

Osteochondrolysis

Nocturnal rectalgia

Aneurysmorrhaphy

Cholecystotomy

Cardio-omentopexy

3.2.1.4 Multiplication de l'éponyme en français

Fibres arquées d'Arnold, fibres en U de Meynert

Flerpès de Bateman, hydroa de Bazin

Maladie d'Osler, maladie de Jaccoud-Osler

Greffe de Reverdin, greffe de Thiersch

Hémoblaste de Sabrazès, myéloblaste de Schridde et Naegeli

Opération de Sourdeville, opération de Lempert

Hyperkératose congénitale de Unna, hyperkératose ichtyosiforme de Darier, hyperépidermatrophie généralisée de Vidal

\author{
Tentorial nerve \\ Thalamic radiation \\ Foxhole arthritis \\ Pulled elbow \\ Gum rash \\ Lead line \\ Stege \\ Paratrooper fracture \\ Trigeminal impression \\ Herald patch \\ Main bundle \\ Signet ring cell \\ Straight leg raising test \\ Prickle cell layer \\ Acute fulminating pyodermia \\ Infraclavicular triangle \\ Fibrolipoma \\ Ventral sensory nucleus \\ Hepatic cords \\ Herniation of nucleus pulposus \\ Neurodocitis \\ Pectunculus \\ Pelvic colon \\ Migrating phlebitis \\ Lingula \\ Tactile field \\ Supraventricular crest \\ Compact layer
}




\title{
3.2.2 Formes composées
}

3.2.2.1 Maintien du déterminé et suppression du déterminant éponymique en anglais

Acrodermatite entéropathique de Danboldt-Closs

Acrodermatitis enteropathica

Patella partita de Wenzel-Gruber

Patella partita

3.2.2.2 Maintien du déterminé et substitution du déterminant éponymique en anglais

Syndrome d'Apert-Cooke-Gallais

Fracture d'Olivier-Lenoir

Image de Purkinje-Sanson

\author{
Adrogenial syndrome \\ Boxer's fracture \\ Mirror image
}

\subsubsection{Transformation de tous les éléments en anglais}

3.2.2.3.1 Par explicitation du déterminé maladie, syndrome, etc.

Maladie d'Alibert-Bazin

Maladie de Derrick-Burnet

Maladie de Jaccoud-Osler

Opération de Lawson-Tait

Syndrome de Marie-Leri

Maladie de P. Marie et Sainton

Maladie de Petges-Cléjat

3.2.2.3.2 Par un procédé plus élaboré

Mur de Schwartze et Eysel

\author{
Arthropathia psoriatica \\ Query fever \\ Subacute bacterial endocarditis \\ Cholecystostomy \\ Opera-glass hand \\ Cleidocranial dysostosis \\ Poikilodermatomyositis
}

Tegman mastoideum

\section{CONCLUSION}

Cet essai de classification confirme la confusion qui règne dans le domaine de l'éponymie médicale et met en lumière les difficultés qui attendent le traducteur. Celuici ne pourra donc que souscrire aux souhaits et aux mises en garde des médecins euxmêmes qui se plaignent du phénomène. " Il nous faut être beaucoup plus sévères dans la lutte contre les éponymes " déclarait naguère le Dr J.C. Sournia, même si, faute de pouvoir rayer tous les noms propres du lexique médical, il faut se résigner à en conserver quelques-uns parmi les " inextirpables". Pour le reste, "les éponymes passent, les maladies et les symptômes restent ", notait encore le Dr Sliosberg, "et il serait à souhaiter que les éponymes soient remplacés par des qualificatifs plus précis et plus universellement admis ${ }^{5}$.

Notes

1. SLIOSBERG, A. (1970) : "Quelques considérations sur la traduction médicale et pharmaceutique", Traduire, $\mathrm{n}^{\circ} 63$, juin, Paris.

2. VAN HOOF, H. (1959) : "Réflexions sur le langage médical ", la Presse médicale, 67, no 11, février, Paris.

3. VAN HOOF, H. (1960) : "La traduction médico-pharmaceutique ", Lebende Sprachen, $\mathrm{n}^{\circ}$ 5, Berlin.

4. VAN HOOF, H. (1970) : "La traduction médico-pharmaceutique ", le Langage et l'homme 4, $\mathrm{n}^{\circ}$ 12, Bruxelles; Meta, 15, $\mathrm{n}^{\circ} 2$, Montréal.

5. SOURNIA, J.C. (1974) : Langage médical moderne, Hachette, 118 p. 